\title{
Diagnóstico y Vulnerabilidad Socio-Territorial en la VI Región
}

\section{Diagnosis of Socioterritorial Vulnerability in the VIth Region}

\author{
Germán Rozas O.* \\ Víctor Martínez; \\ Daniela Bolivar; \\ Edison Leiva; \\ Egidio Céspedes.
}

\begin{abstract}
Resumen
El presente artículo es fruto de una Investigación Fondecyt. El objetivo central del estudio es contribuir a la caracterización de la coordinación actual de las políticas públicas orientadas a la superación de la pobreza, en la perspectiva de conformar un modelo de intervención y gestión de metaredes en desarrollo social.

En dicho contexto, el propósito de esta publicación es dar cuenta del Diagnóstico de la VI Región (Chile) haciendo uso de una metodología, que incorpora el Diagnóstico de Dinámicas. En esta línea de acción se presenta un nuevo indicador social que hemos denominado Indicador de Vulnerabilidad Socio-Territorial.
\end{abstract}

Es necesario indicar además que este trabajo se enmarca dentro del área de la Psicología Comunitaria, como un aporte al ámbito de la construcción de Política Social.

Palabras Claves: Superación de la Pobreza, Redes Institucionales, Diagnóstico de Dinámicas.

\begin{abstract}
This paper is one product of a Fondecyt's research. The principal objective in this study is tray to buil a description of actual coordinality of the publics policity to guide the overcoming of poverty, in the way to make a model of action and managment of nets on social developped.

In this topic, the goal of this publication is show a diagnostic concerning to region's sixth (Chile) using one type of methodology, which name is Dinamic's Diagnostic. In a another hand of this paper, it is showing a new social's indicator that it was calling Index of Social-Territorial Vulnerability.

It is need to say that this work belong to Community Psychology's area, in the way to make a contribution to Social Policity.

Keys Words: Overcoming of Poverty, Institucional“s Nets, Dinamic"s Diagnostic.
\end{abstract}

* Psicólogo Comunitario, Magister Universidad Libre de Bruselas, Docente Universidad de Chile. grozas@uchile.cl 


\section{Marco de Referencia}

La discusión actual acerca de las políticas sociales, la reformulación del rol del Estado, el fortalecimiento de la sociedad civil y el mercado, han generado un conjunto de visiones y experiencias que, con distintas intensidades, están reconfigurando el cómo la sociedad chilena promueve el desarrollo social y orienta su acción hacia la superación de la pobreza. En este sentido, se asume que actualmente las políticas sociales se encuentran en crisis. Las conceptualizaciones subyacentes a las políticas sociales ya no parecen ser pertinentes a la realidad social; la efectividad e impacto de programas sociales están por debajo de sus potencialidades más evidentes y la inserción de criterios y recursos privados en el diseño de planes y programas sociales aún no responden a una acción coordinada, generando un escenario bastante autoreferente y fragmentado desde la perspectiva de los grupos sociales en situación de pobreza. Como lo señala el PNUD (1998), se aprecia una contradicción entre el crecimiento objetivo del país y la subjetividad de la gente.

El Estado Chileno ha considerado permanentemente el tema de la pobreza como una de sus urgencias centrales para el desarrollo social del país. Las políticas públicas elaboradas para tal propósito, en general, han partido de una teoría o de un concepto de la pobreza que a la mirada de hoy día resulta restringido. Dicho concepto se centra en las carencias, en los déficits de las personas y familias pobres. La proyección de las políticas sociales a partir de esta lógica se orienta, en su esencia, a subsidiar el complemento de aquellas necesidades insatisfechas en los pobres. Esta forma de paliar la pobreza es coherente con la estructura del Estado. Este último se constituye históricamente en una especie de diferentes edificios sociales de rango ministerial separados unos de otros. Esta paradójica desvinculación estructural en un
Estado centralizado es coherente con el enfoque de la pobreza centrado en las carencias. Esta asociación implícita se encuentra hoy día en cuestionamiento, en tanto la pobreza se hace crónica y el Estado es catalogado de ineficaz en el plano de las políticas sociales (Banco Mundial, 1997).

De ahí que la CEPAL (1992) ha identificado distintas dimensiones esenciales en la relación entre el sector público y los diferentes actores sociales, que constituyen el fundamento de la propuesta de Transformación Productiva con Equidad, la promoción de iniciativas que involucren una multiplicidad de instituciones públicas en torno de programas intersectoriales de amplia cobertura, expresando con esto, la necesidad de enfocar integralmente el fenómeno de la pobreza, dando cuenta y al mismo tiempo abarcando la multiplicidad y diversidad de situaciones relacionadas con ella. (Banco Mundial, 1997)

Frente a la crítica conceptual sobre la pobreza centrada en las carencias surge desde el mismo Estado y otros ámbitos el concepto de "Superación de la Pobreza". La perspectiva en este sentido es hacia el "empoderamiento" de la población, hacia la acciones de asociacionismo, al cambio de actitud de actores privados, la capacidad gerencial de los organismos públicos, etc. (Fosis, 1993).

Las proyecciones de hoy en día centradas en la superación nos dirigen también al concepto del Capital Social. Se trata de potencialidades, de habilidades, de recursos en los grupos sociales, en la cultura, en las personas, en la familia. Se hace eco de la presencia de acciones compartidas, con generación de nuevas oportunidades, a través de la apertura del mundo empresarial a la diversidad social.. Todos son aspectos que apelan a una mirada integral del desarrollo social y son sinónimos del Capital social. (Kliksberg, 1998). 


\section{Pobreza Moderna y Pobreza por Atraso}

Para una mejor comprensión conceptual de la terminología utilizada en este artículo, a continuación una breve descripción de Pobreza Moderna y por Atraso.

Desde un acercamiento cualitativo al tema de la pobreza, recogido en los trabajos de CELADE a partir de los años ' 80 , es posible distinguir dos perfiles para identificar modalidades en que ésta puede contextualizarse según la dinámica de desarrollo en la cual se generan los fenómenos de carenciación.

Por pobreza moderna puede entenderse aquella situación de carencia en la cual se ven implicados segmentos más o menos significativos de población, insertos en un esquema socioeconómico caracterizado por fuertes procesos modernizadores que hacen obsoletas las antiguas relaciones de producción y que tienen como consecuencia la marginación de dichos sectores en virtud de su incapacidad de incorporarse a las nuevas tecnologías o a las nuevas redes de intercambio de bienes y servicios. Es frecuente identificar este fenómeno en los márgenes de las sociedades industrializadas; en el contexto latinoamericano, más bien alude a la situación en la cual procesos acelerados de modernización basados en economías con alta concentración de la propiedad, generan una distribución del ingreso fuertemente desigual, dejando a un porcentaje mayoritario de la población por debajo de un nivel mínimo de ingresos.

En cambio, la pobreza por atraso se refiere a personas que pertenecen a segmentos de población que no se han beneficiado de estos fenómenos de modernización, viéndose insertos en economías donde las viejas relaciones sociales de producción son, sin embargo, las predominantes. A escala nacional, son comunidades enteras las que, en virtud de su perfil socioproductivo y a la naturale- za de su precaria inserción en las redes productivas de un aís o de una región, están inmersas en una situación generalizada de atraso económico que afecta a la globalidad de sus estructuras sociales y a su cultura. Es el caso de, por ejemplo, las comunidades indígenas, con sus formas ancestrales de cultivar la tierra, o los mineros del carbón, comunidad sustentada por entero en una explotación que por la naturaleza del mercado de las fuentes energéticas ha ido quedando en desuso.

\section{Metodología}

Para la realización del Diagnóstico como así mismo para la elaboración del Indicador de Vulnerabilidad Socio-Territorial se utilizaron datos extraídos de fuentes de información secundaria. Luego estos datos fueron trabajados bajo la perspectiva del enfoque territorial. Esta línea de análisis territorial es el sustento del diagnóstico realizado.

En cuanto a la elaboración del indicador, los datos emanados principalmente del Censo de 1992 en la VI Región, fueron sometidos al programa computacional Mapinfo, que permitió desarrollar una expresión geográfica de la vulnerabilidad de esta región.

\section{Fuentes de información secundaria}

Las fuentes de las que se obtuvo información para este artículo fueron el Censo de 1992, la encuesta CASEN de 1998 (extraídos principalmente de compendios del Instituto Nacional de Estadísticas -INE- o facilitados por la SERPLAC de la Intendencia de la VI Región), y el Programa Regional de Información Territorial (PRIT6), CD room que reúne información sobre las características demográficas, económicas y te- 
rritoriales del mundo rural de la VI región. Dicho CD fue elaborado por la SERPLAC el año 1999.

\section{Enfoque territorial}

Respecto del enfoque territorial, debemos señalar que su inclusión en el presente estudio se relaciona con la necesidad detectada en la gestión pública de organizar la información acerca de una unidad geográfica sujeto de diseño y planificación de políticas públicas. A partir de 1990, se reemprendió la iniciativa hacia la descentralización mediante nuevos instrumentos y enfoques conceptuales acerca de la gestión de las regiones. Un nuevo cuerpo legal, la Ley Orgánica de Gobierno Interior y Administración Regional (1991), redefinió nuevas instancias para impulsar la desconcentración del poder y de los recursos, iniciativa que ha tenido un proceso lento pero relativamente eficaz a través de la instalación de los Gobiernos Regionales.

Este enfoque asume una perspectiva topológica para la comprensión de las problemáticas sociales, tomadas a partir de su localización y arraigo en una zona geográfica delimitable, además de una mirada igualmente tópica de las posibles soluciones y estrategias de enfrentamiento. Pretende trascender el enfoque estático y parcial que se deriva de revisar dichos fenómenos sólo a partir del acopio de datos estadísticos, que permiten una mirada analítica de la situación pero no su síntesis. Mediante este enfoque se abre la posibilidad de poner en relación los fenómenos de desarrollo y pobreza de una localidad o zona geográfica con los ocurrentes en otras localidades o zonas, sean éstas contiguas o bien remotas.

Por otra parte, el enfoque territorial permite superponer diversas realidades o dimensiones, de modo de relacionar, en una misma localidad o zona geográfica, infor- maciones de diversos órdenes (orografía, hidrografía, suelos, factores productivos, vialidad, población, áreas de pobreza, etc.), permitiendo complementar éstas para una comprensión cabal de las realidades y potencialidades del territorio sometido a revisión.

El enfoque territorial no es un método nuevo, de hecho se utiliza ampliamente en disciplinas tales como la planificación urbana. Sin embargo, resulta novedosa su aplicación para el estudio de los modos de inserción de las políticas sociales, coordinadas intersectorialmente, sobre una unidad territorial con identidad específica.

\section{Diagnóstico de Dinámicas}

El diagnóstico de dinámicas es un método que tiene como objetivo facilitar la identificación de procesos (sistemas) relacionados con el desarrollo y la selección de "palancas" o estrategias de intervención que sean pertinentes para superar la pobreza en localidades, territorios y localidades específicas.

El diagnóstico de dinámicas contempló cuatro pasos fundamentales. El primero fue la caracterización de las variables sociales, de las condiciones del territorio y de su entorno. Esta caracterización se construyó a partir de los antecedentes estadísticos disponibles en el Sistema de Información Regional (SIRIG) de la VI región y el Instituto Nacional de Estadísticas (INE) sobre demografía, nivel educacional, pobreza, salud, ocupación y desocupación. Estos antecedentes permitieron ir diferenciando las comunas según sus distintos "perfiles", ya sea en términos de nivel educacional, actividad laboral, recursos productivos y accesibilidad vial.

El segundo paso del diagnóstico de dinámicas consistió en identificar, analizar y establecer relaciones entre los diversos problemas que afectaban a los grupos de interés para nuestro estudio (sectores de pobreza) 
en función de un problema central: el mecanismo de reproducción de la pobreza. Bajo esta mirada, y mediante la metodología del diagnóstico de dinámicas, se analizó cómo las características comunales y la relación entre sus distintos "perfiles" explicaban la existencia de la pobreza, considerando no sólo los antecedentes del Censo, sino también los recursos y características geográficas de cada sector. La relación y cruce de todos estos antecedentes permitieron dar cuenta de la existencia y mantención de la pobreza en las distintas comunas de la VI región.

El tercer paso del diagnóstico de dinámicas consistió en buscar un conjunto de objetivos posibles de alcanzar y que permitieran revertir los problemas antes identificados. Específicamente, este estudio consideró aquellos problemas posibles de transformar para prevenir la producción de la pobreza, aprovechando los recursos de su entorno y su capital social.

El cuarto y último paso del diagnóstico de dinámicas fue el análisis de sistemas y estrategias o palancas de intervención, es decir, la identificación de procesos donde existiesen estrategias claves o "palancas" que permitieran generar desarrollo en la comuna o en la región. Esto implicó formular una propuesta de intervención con un carácter "estratégico" en tanto fuese capaz de producir efectos positivos en aquellos procesos que se relacionaran con la mantención y reproducción de la pobreza. Desde este punto de vista, esta investigación generó una propuesta para la superación de la pobreza cuya novedad consistió en un modelo que integró los conceptos de intersectorialidad y diagnóstico territorial.

Los dos últimos pasos no están presentes en este artículo, se requiere un mayor acopio de información, que aún no se ha alcanzado.

\section{Antecedentes de la Provincia de Cachapoal, VI Región}

El diagnóstico se realizó en las tres provincias Cachapoal, Colchagua y Cardenal Caro, de la VI Región. Pero por limitaciones de espacio se presenta a continuación sólo la provincia de Cachapoal. Sin embargo al final del artículo las conclusiones presentadas corresponden a las tres provincias de la Región.

\section{a) Características de la población}

La población de la provincia de Cachapoal corresponde al $69 \%$ de la población de la VI región. El 39\% de esta población vive en Rancagua, mientras que el resto se concentra mayoritariamente en Rengo $(9,1 \%)$, San Vicente $(7,4 \%)$ y Machalí $(5,1 \%)$.

La población de la provincia de Cachapoal es principalmente urbana, representando el $71 \%$ de la población total. La población restante (29\%) corresponde al sector rural. Además, la provincia concentra el mayor porcentaje de habitantes en zona urbana y rural de la VI región (alrededor de los 340.000 y 140.000 habitantes respectivamente).

En relación a la población por comunas, ésta oscila entre 5 mil y 35 mil habitantes a excepción de Rancagua, la cual tiene una población aproximada de $190 \mathrm{mil}$.

\section{Población rural:}

De la provincia de Cachapoal, las comunas que aportan el mayor porcentaje de población rural son San Vicente, Rengo y Coltauco. Sin embargo, las comunas con mayor proporción de población rural son Coltauco (88\%), Pichidegua (74\%), Las Cabras (66\%), Malloa (64\%) y Codegua (64\%).

De las 17 comunas de Cachapoal, 9 tienen una población rural que alcanza más del $50 \%$. de la población comunal. 
Población urbana:

En relación a la población urbana, las comunas que aportan mayor población urbana a la provincia son las comunas de
Rancagua, Rengo y Machalí. Sin embargo, las comunas que concentran una mayor proporción de población urbana son Rancagua (96\%), Machalí (90\%), Graneros (79\%), Doñihue (78\%) y Mostazal (76\%).

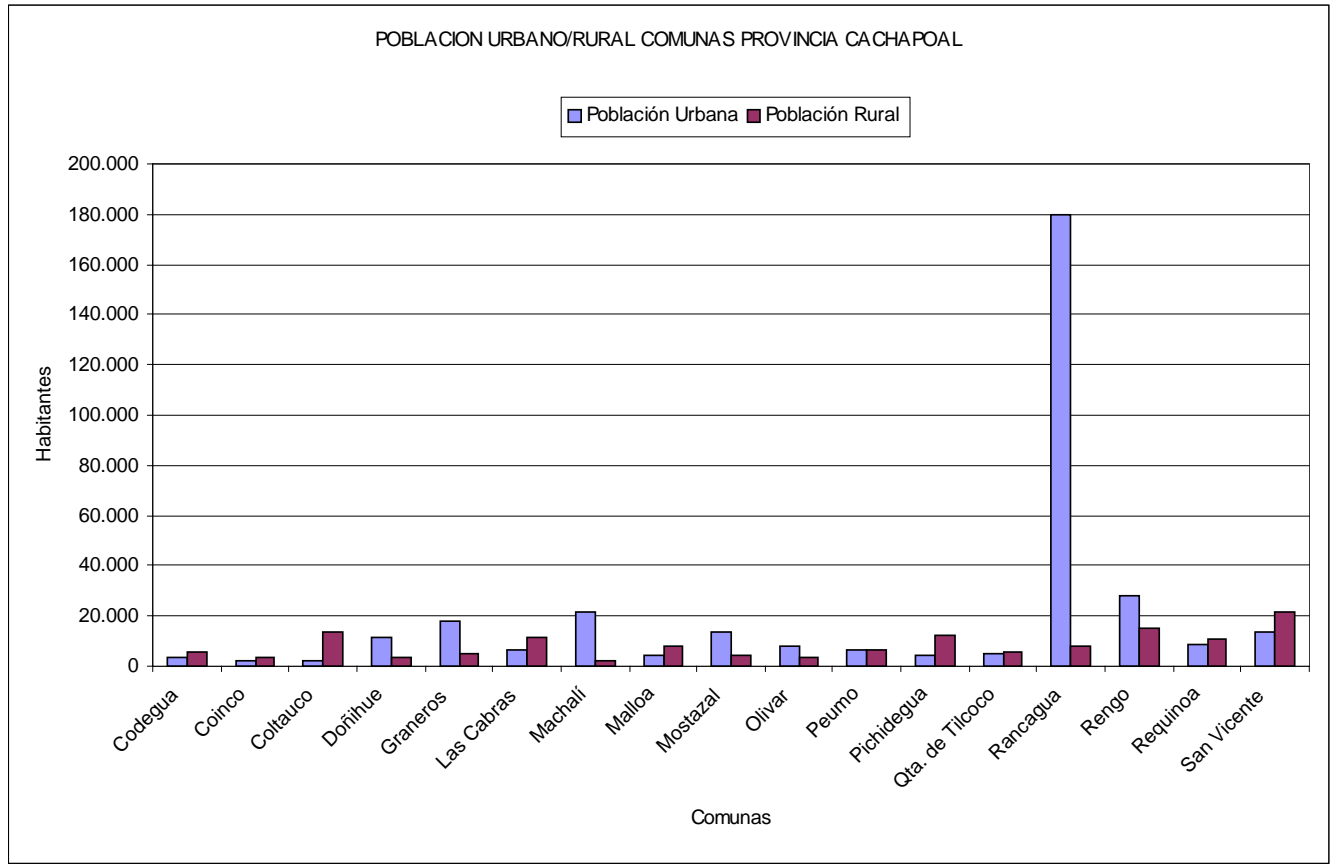

Fuente: Censo 1992

\section{Densidad.}

La comuna más densa es Rancagua (720 $\mathrm{hab} / \mathrm{km} 2)$. Le sigue Olivar (254 hab/km2), Graneros (199 hab/km2) y Doñihue (186 $\mathrm{hab} / \mathrm{km} 2$ ). Machalí, a pesar de ser una de las comunas más habitadas de la provincia de Cachapoal, tiene una densidad baja, pues la mayor parte de su territorio coincide con la cordillera de los Andes.

\section{Migrantes}

La población migrante de la provincia se concentra principalmente en la comuna de Rancagua (30,2\%). Sin embargo, las comunas que tienen mayor proporción de migrantes en relación a su propia población son las comunas de Machalí (18\%), Olivar (16\%), Doñihue (15\%) y Codegua (14\%).

\section{Minorías étnicas}

A nivel provincial, la mayor cantidad de grupos étnicos se encuentra en Rancagua, pero es Machalí la comuna que concentra un porcentaje mayor en relación a la población Comunal (8\%). Le sigue Graneros $(7,5 \%)$ y Rancagua (7\%).

\section{b) Pobreza}

La provincia de Cachapoal concentra el $63 \%$ de las personas con necesidades básicas insatisfechas (NBI) de la VI región, esto 
porque es la provincia que concentra la mayor cantidad de población. Por el mismo motivo, Rancagua es la comuna que tiene la mayor cantidad de pobres de la provincia, alrededor de 50 mil personas, que equivalen al $26 \%$ de la provincia.

$\mathrm{Al}$ interior de la provincia de Cachapoal, el $41 \%$ de sus habitantes tiene sus necesidades básicas insatisfechas, mientras que el $58 \%$ no los tiene.
Las comunas de Cachapoal, por su parte, presentan importantes porcentajes de población con necesidades básicas insatisfechas, ya que de las 17 comunas de la provincia de Cachapoal, 12 de ellas tienen, en relación a su propia población, un porcentaje de personas con NBI superior al $50 \%$ :

\begin{tabular}{|l|c|}
\hline Comuna & Porcentaje Habitantes con NBI \\
\hline Coinco & $63,7 \%$ \\
\hline Olivar & $62,7 \%$ \\
\hline Doñihue & $62 \%$ \\
\hline Qta. De Tilcoco & $61,3 \%$ \\
\hline Mostazal & $59,8 \%$ \\
\hline Codegua & $58,8 \%$ \\
\hline Requinoa & $55,4 \%$ \\
\hline Las Cabras & $55,4 \%$ \\
\hline Malloa & $54,2 \%$ \\
\hline Coltauco & $53,1 \%$ \\
\hline Pichidegua & $51,1 \%$ \\
\hline Machalí & $50,1 \%$ \\
\hline
\end{tabular}

Fuente : Censo 1992

Por otro lado, las comunas con mayor porcentaje de personas sin NBI son Rancagua (73\%), Rengo (57\%), San Vicente (56\%), Graneros (55\%) y Peumo (54\%).

Esta proporción se invierte si es que se toma como referencia el total de la población provincial. En este caso, Rancagua (26\%), Rengo (9\%) y San Vicente (8\%) concentran la mayor cantidad de población con NBI de la Provincia en su conjunto:

Según la encuesta CASEN (1998), Rancagua es la comuna de la provincia de Cachapoal que reúne la mayor cantidad de pobres e indigentes de la región. En segundo lugar le sigue Rengo. En términos de indigencia, Rancagua concentra el 35\% de los indigentes de 13 comunas de la región. Le sigue Rengo con el 14\%. En términos de pobreza no indigente, el orden se mantiene, encontrándose Rancagua en la cabeza con un $32 \%$ y siguéndolo Rengo con un $9 \%$. Estos datos han sido calculados según la población total de la región.

\section{c) Jefatura de hogar femenina}

En la provincia, sólo el $21 \%$ de los hogares tienen como jefatura a una mujer. Encabezan esta lista Rancagua (24\%), Machalí (24\%) y Olivar (23\%). Las demás comunas se mantienen en un rango promedio de $18 \%$ aproximadamente. 


\section{d) Educación}

A nivel regional, la provincia de Cachapoal tiene el promedio de años de estudio más alto (8 años). La comuna con un promedio más alto es Rancagua. Sus habitantes alcanzaron en el Censo de 1992 un promedio de años de estudio que superaba los 9 años. El segundo promedio más alto se encuentra en Machalí (8,1 años) y el tercero en Graneros ( 7,8 años)

Por otra parte, las comunas con promedio más bajo de años de estudio son Las Cabras $(6,2)$, Pichidegua $(6,2)$, Coltauco $(6,4)$ y Codehua $(6,6)$.

En la provincia, la población analfabeta alcanza el 5,46\% de la población. De esta, el $51 \%$ es urbana y el $49 \%$ es rural. En el sector urbano, las comunas con mayor porcentaje de analfabetismo son Coltauco (7\%), Las Cabras (6,5\%), Pichidegua (6,5\%), Quinta de Tilcoco $(6 \%)$ y Coinco (6\%). En el sector rural, se encuentran Las Cabras (13\%), Pichidegua (11\%), Coltauco (10\%) y Mostazal (10\%).

Estos antecedentes indican que las comunas de Las Cabras, Pichidegua y Coltauco aparecen como las tres comunas con mayores problemas educacionales de la provincia.

En relación a la cantidad de matrículas escolares que ofrece la educación municipalizada y particular-subvencionada, el $67 \%$ de las matrículas que existen para la población en edad escolar corresponden a la educación básica, mientras que la educación media representa $18 \%$. El resto (15\%) corresponde a la educación preescolar.

En relación a la educación media, sólo el $18 \%$ de la oferta total de matrículas municipales y subvencionadas en la provincia de Cachapoal corresponden a la educación secundaria, donde la oferta de enseñanza media científico humanista corresponde al $13 \%$ y sólo el $5 \%$ a la educación técnica profesional.
Las estadísticas permiten dilucidar, por lo tanto, que la oferta educacional se ha concentrado preferentemente en la educación básica: en prácticamente todas las comunas de la Provincia de Cachapoal, la educación básica corresponde entre el 66 y el $75 \%$ de las matrículas municipalizadas y particular subvencionadas, sobresaliendo en un extremo la comuna de San Vicente (sólo 31\%) y en el otro Malloa (90\%).

En relación a la enseñanza media la realidad es diferente. En tres comunas no existen matrículas en la educación científico humanista (Malloa, Olivar, San Vicente) y en otras no existen matrículas en educación técnico profesional (Codegua, Coinco, Doñihue, Las Cabras, Machalí, Malloa, Olivar, Pichidegua, Quinta de Tilcoco y San Vicente) ni en la educación municipal ni en la subvencionada.

Por otra parte, las comunas que ofrecen más matrículas en la Enseñanza Media Científico-Humanista son Rengo (18\%), Rancagua (17\%) y Codegua (15\%), mientras que las comunas que ofrecen más matrículas en la enseñanza Técnico-Profesional son las comunas de Coltauco (16\%), Rancagua $(8 \%)$ y Olivar $(8 \%)$.

\section{e) Desocupación}

La Provincia de Cachapoal aporta la mayor cantidad de desempleados a la VI región, con una tasa de 8,91. Esto tal vez por ser la provincia que concentra la mayor cantidad de población. Del total de la población provincial, el $9 \%$ de la población económicamente activa se encuentra desempleada. El $91 \%$ restante se desenvuelve en actividades de diversa naturaleza.

$\mathrm{Al}$ interior de la provincia, la comuna que concentran una mayor cantidad de desempleados es Rancagua. En segundo lugar se encuentra Rengo, luego San Vicente y posteriormente Machalí. La comuna con menos desempleados es Coinco. 


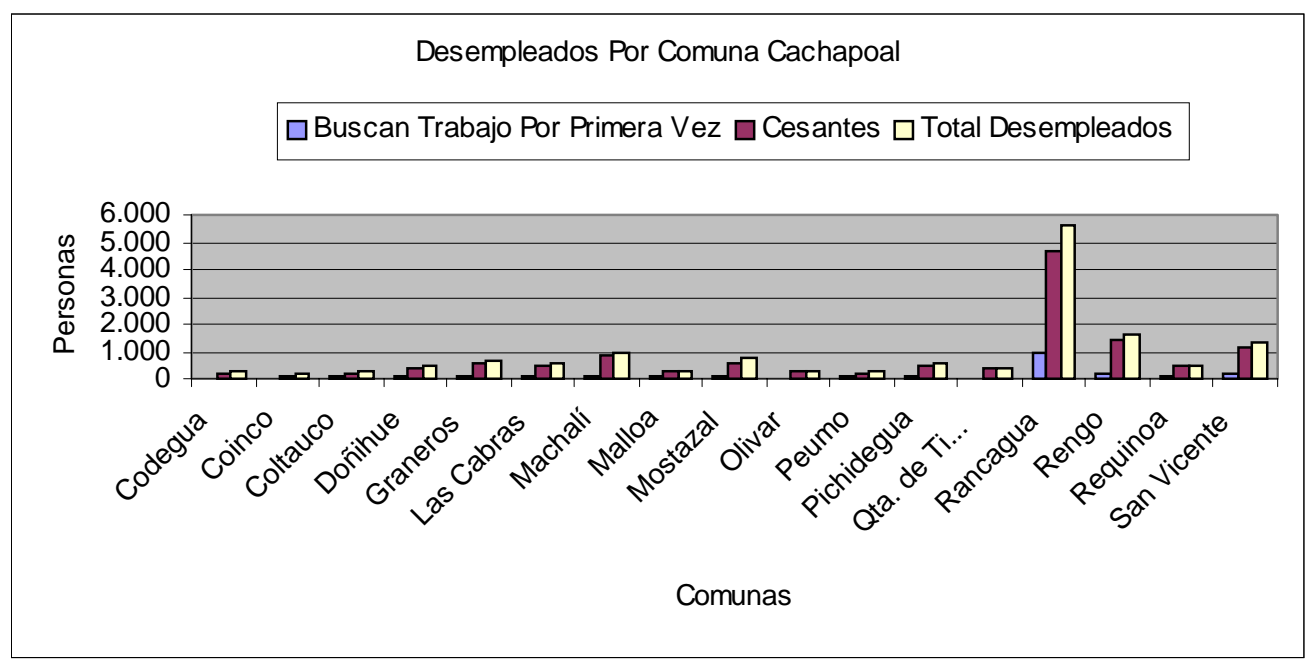

Fuente: Censo 1992

Según la proporción comunal, la comuna que tiene mayor proporción de desempleados es Mostazal (con una tasa de cesantía de $12 \%$ ), le siguen Quinta de Tilcoco (tasa: 10,82$)$, San Vicente $(10,79)$ y Machalí $(10,76)$.

\section{f) Perfil laboral}

En la provincia de Cachapoal el 35\% de su población es económicamente activa. De este porcentaje, en el año 1992 el 8,91\% estaba desempleada (se encontraba cesante $o$ buscaba trabajo por primera vez) y el $91,09 \%$ constituía la fuerza de trabajo de la región.

En relación a los índices de desempleo, las comunas con mayores índices son, según el Censo de 1992, Mostazal (12,03\%), Quinta de Tilcoco $(10,82 \%)$, San Vicente de Tagua Tagua (10,79\%), Machalí (10,76\%) y Rengo $(10,16 \%)$. Le siguen las comunas de Doñihue $(9,98 \%)$, Pichidegua $(9,90 \%)$, Coinco $(8,88 \%)$ y Rancagua (8,49\%).

En relación a las actividades productivas, la actividad agropecuaria es una de las más importantes. El $30 \%$ de las personas en edad económicamente activa de la provincia de Cachapoal se dedican a la agricultura. De este, el $85 \%$ se dedica a los cultivos en general y cultivos de productos de mercado. Más específicamente, 8 de las 17 comunas de Cachapoal, tienen más del $50 \%$ de su población económicamente activa realizando actividades agrícolas. Sobresalen Pichidegua (63\%) (que en su mayoría se preocupa por los cultivos en general), Codegua (63\%), Coltauco (59\%), Las Cabras (55\%), Peumo (53\%), Requinoa (53\%), Malloa (52\%) y Qta. De Tilcoco (51\%).

La segunda actividad más importante a nivel provincial es el comercio, con un $15 \%$. En este rubro, la comuna más importante de la provincia es Rancagua (20,3\%). Le sigue Requinoa (17\%), Olivar (15\%), San Vicente $(14,4 \%)$ y Coltauco $(14,4 \%)$.

La tercera actividad más importante de la provincia es la industria $(9,7 \%)$, donde sobresalen las comunas de Doñihue $(23,45 \%)$, y las comunas de Coinco, Malloa, Mostazal, Quinta de Tilcoco y Rengo con porcentajes alrededor del 15\%.

La cuarta actividad de la provincia es la minería (7\%). Esta actividad se concentra 
principalmente en Rancagua y Machalí donde trabaja en este rubro un $14 \%$ y un $13 \%$ (respectivamente) de su población económicamente activa.

La quinta actividad es el transporte, almacenamiento y comunicaciones (6\%), la cual es encabezada por las comunas de Olivar $(7,8 \%)$, Rancagua $(7,65 \%)$, Requinoa (6\%) y Graneros $(5,8 \%)$.

\section{Resultados: Diagnóstico de Dinámicas de la Provincia de Cachapoal}

Las características demográficas, productivas y sociales relatadas en este informe permiten dilucidar que cada comuna tiene características que le dan un perfil distintivo, dado por sus antecedentes poblacionales y su perfil educacional y productivo. Al graficar estos antecedentes, es posible observar que los perfiles de algunas comunas son similares entre sí, los cuales en la gran mayoría coinciden con comunas colindantes territorialmente. Siguiendo este criterio, llamado para este efecto "perfiles socio-productivos", las comunas fueron agrupadas según sus similitudes, en tres "sectores" diferentes, las cuales se describirán a continuación:

\section{a) Sector agrícola tradicional}

-Su perfil educacional es de baja escolaridad con presencia de altos niveles de analfabetismo. La oferta de educación municipalizada y particular subvencionada es preferentemente básica, baja oferta de enseñanza media científico-humanista y prácticamente nula en la enseñanza técnico-profesional.
Su perfil laboral está dado preferentemente por el trabajo agrícola, especialmente de productos de mercado y horticultura a través de empresas que van desde las microempresas familiares hasta grandes empresas. También hay en ellas un número considerable de microempresas familiares en el rubro comercial.

Estas comunas disponen de frutales, aunque no concentran la cantidad más importante de la provincia. Cuentan con algunas pequeñas propiedades mineras nuevas que contemplan minas de oro y cobre. El cultivo forestal no es importante. Según los mapas, el acceso a Las Cabras y Peumo no se hace difícil producto de la presencia de un camino pavimentado que las atraviesa. Sin embargo, da la impresión que sectores importantes de Las Cabras, Coltauco y Codegua quedan inaccesibles por la vía del transporte.

Por lo tanto, la pobreza existente en ese sector es una pobreza rural asociada más bien a la marginalidad y a la falta de preparación técnica que impide hacer un buen uso de los recursos. La pobreza se explicaría en la medida que el sector agrícola se mantiene al margen, ya sea por inaccesibilidad vial o de otro tipo, de los servicios educacionales, lo cual implicaría un perfil no sólo bajo en escolaridad sino también poco especializado en el recurso que tienen más a mano: la producción agrícola. Además el perfil laboral no siempre da cuanta de un aprovechamiento de todos los recursos naturales que existen en la zona. Todo lo anterior parece indicar que se trata de un sector que genera un tipo de pobreza por atraso.

Gráficamente, los antecedentes parecen indicar que se da el siguiente proceso: 


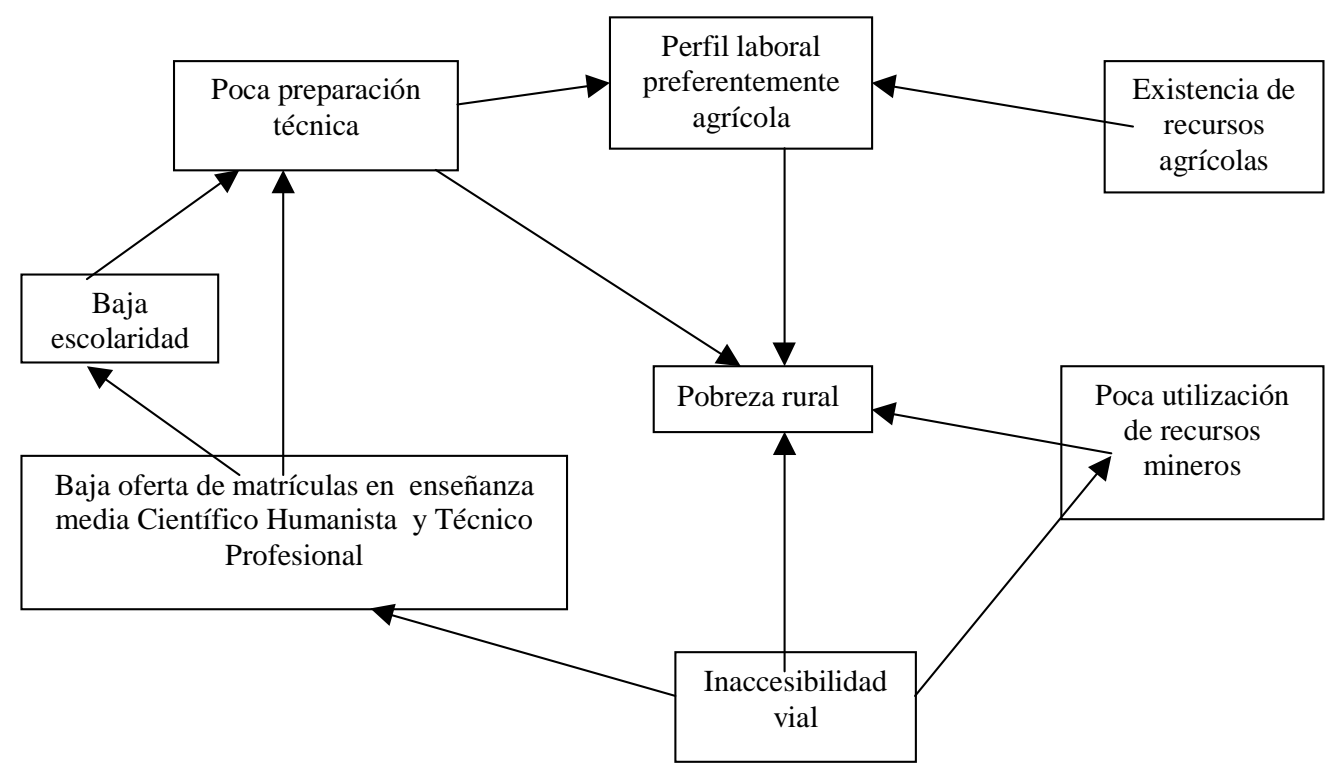

\section{b) Sector semiagrícola}

Un segundo grupo de comunas que reúnen características similares son las comunas de Doñihue, Olivar, Quinta de Tilcoco y Coinco. Su principal característica es que ellas tienen las proporciones más alta de pobreza de la provincia (sobre el 60\%).

En ellas, la distribución población rural y urbana es más equitativa (con cierta tendencia hacia la población urbana) y su densidad es más bien alta en comparación al resto de las comunas de la provincia.

Su perfil laboral está dado por un trabajo preferentemente agrícola pero que no alcanza a superar en todos los casos el $50 \%$. El trabajo en las minas es muy poco, excepto en Olivar en donde el 3\% de su población se dedica a este rubro. Sin embargo, su población se dedica en porcentajes mayores al trabajo en industrias manufactureras (entre un 7,6 y un $23,4 \%$ ). La población que se dedica al sector construcción es relativamente bajo (desde un 1 a un 3,7\%), en tanto la población que trabaja en el comercio asciende entre un 8 y un $14 \%$. Olivar destaca por tener el porcentaje más alto de la provincia que se dedica al rubro de transportes $(7,8 \%)$.
Su perfil educacional está dado por una población que en promedio cursa 6,9 años de estudio (un año menos que el promedio provincial) y con un promedio relativamente alto de analfabetismo que no alcanzan a ser los más altos de la provincia (cerca del $5,8 \%$ de analfabetismo urbano y alrededor del $8 \%$ del analfabetismo rural). La oferta de enseñanza básica alcanza un porcentaje importante, pero la oferta de matrículas en la enseñanza media científico humanista es en promedio el 9\% del total de matrículas. En este caso sobresale la comuna de Olivar que no cuenta con ninguna matrícula en enseñanza media científico-humanísta en establecimientos municipales ni subvencionados. En relación a la enseñanza media técnicoprofesional, ninguna de las cuatro comunas ofrecen matrículas en este tipo de educación, ni en escuelas municipalizadas ni en particular-subvencionadas.

Aún encontrándose al "centro" de la provincia (valle central), este grupo de comunas no cuentan con grandes concentraciones de frutales o productos agrícolas. Olivar es la más favorecida, sin embargo en Coltauco se observan escasos recursos agropecuarios aunque un poco más en cultivos forestales. 
En relación a los recursos mineros, en Olivar y parte de Coltauco existen pequeñas propiedades mineras nuevas. Además en ellas se desarrolla alguna actividad minera en cobre y en menor proporción, oro.

$\mathrm{El}$ acceso vial es aparentemente bastante adecuado. Los mapas dan la impresión de ser comunas bastante accequibles a través de la vía terrestre.

Por otro lado, estas comunas poseen una cantidad importante de atractivos culturales, de hecho concentran junto con Malloa, la mayor cantidad de atractivos culturales de la provincia.
En conclusión, los antecedentes del Censo de 1992 indican que en estas comunas hay una tendencia hacia la urbanización y que, por este motivo, su perfil laboral es más heterogéneo: a pesar que el trabajo agrícola es lo más importante, aparecen también otras actividades como la industria y el comercio. Simultáneamente, su perfil educacional se mantiene bajo y parece no contar con territorio adecuado para el desarrollo agrícola.

Gráficamente, la situación podría ilustrarse como sigue:

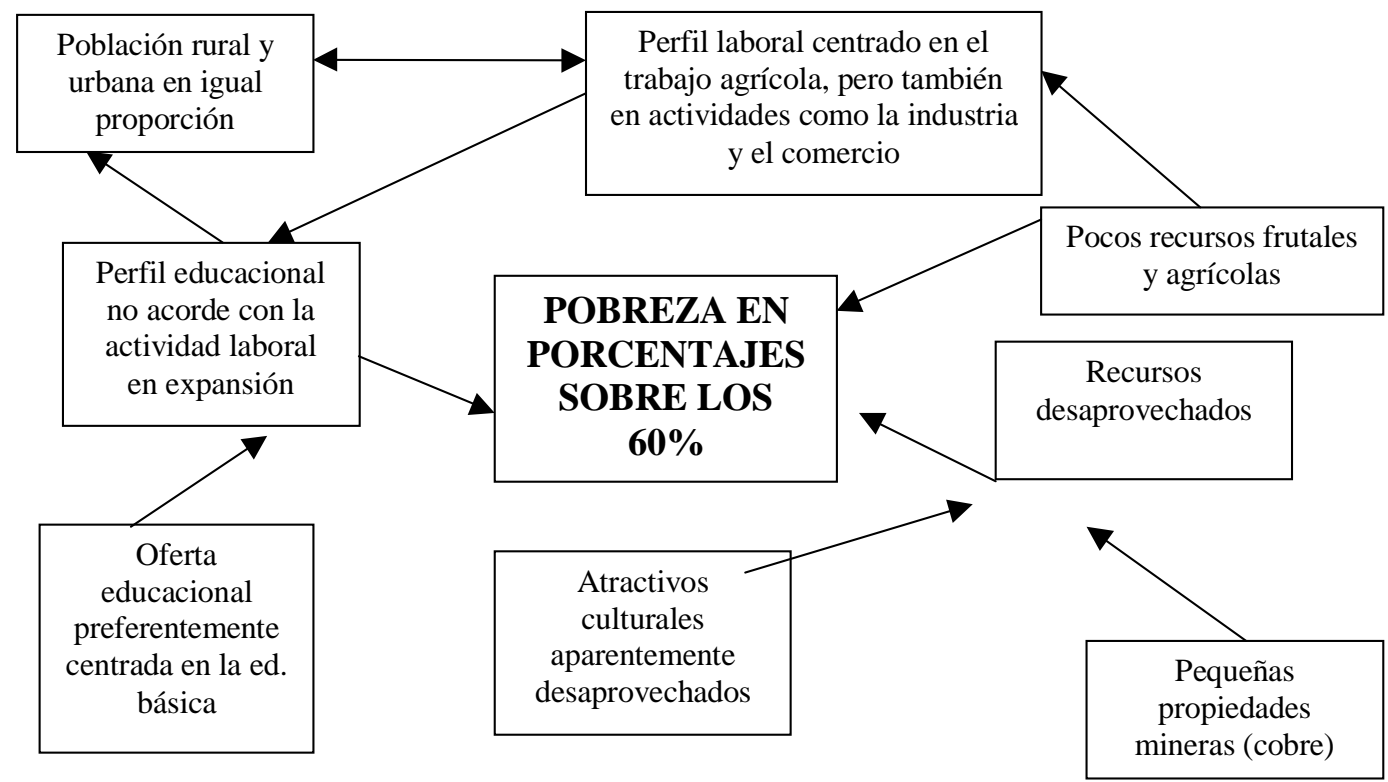

De esta forma, la pobreza se explicaría por un perfil educacional no acorde con las exigencias laborales (baja preparación para actividades económicas como el comercio y la industria) y por recursos naturales y productivos desaprovechados.

\section{c) Sector urbano}

En otra situación se encuentran las comunas de Rancagua, Graneros y Machalí. Estas son un foco de desarrollo de la provincia, ya que en ellas se concentran la mayor cantidad de población, concentran la mayor proporción de población urbana, son más densas en su distribución, concentran mayor cantidad de migrantes y de población indígena.

Su perfil laboral se define, a diferencia de las otras comunas, por tener las proporciones más alta de la provincia en los rubros de comercio, hotelería, minería, construcción $\mathrm{y}$ transporte. Cuenta con un porcentaje muy pequeño de trabajo agrícola. En particular en la comuna de Rancagua, se concentran una importante cantidad de empresas agropecuarias, mineras y de comercio, ya 
sean familiares, formales, pequeñas, medianas y grandes empresas.

El perfil educacional de estas comunas se caracteriza por tener los tres promedios de años de estudio más altos de la provincia (entre 7,8 y 9,2 años). Además, la población analfabeta de las comunas se encuentra cercana al promedio provincial. La educación básica ocupa, al igual que en el resto de las comunas, la mayor proporción de la oferta educacional. Sin embargo, en estas comunas también existe oferta en enseñanza científico humanista y técnico profesional. En el primer caso, las comunas en cuestión tienen una oferta que en proporción no coincide necesariamente con los mayores porcentajes de la comuna, sin embargo, la oferta es significativa (12,8\% Graneros, 8,4\% Machalí y 16,8\% Rancagua). En el segundo caso, estas comunas coinciden con ser dos de las cuatro comunas que tienen enseñanza técnico profesional en la provincia (excepto Machalí, que no tiene matrículas en este tipo de enseñanza).

En relación a sus recursos, estas comunas se encuentran en una ubicación privilegiada de la zona en términos de cultivos de frutales y recursos mineros. En ellas existen grandes propiedades mineras antiguas y nuevas (que comparten también con las comunas de Codegua y san Franciso de Mostazal), y extracción de minerales de cobre y plata. Cuenta con pocos recursos forestales, una adecuada red vial, atractivos culturales y naturales. En Rancagua se concentra una importante cantidad de la oferta educacional de la provincia, especialmente la oferta privada.

En este sector (sector urbano), la pobreza puede explicarse por la generación de polos de desarrollo que facilitaron la emigración de la población de la VI región hacia estas comunas (Rancagua, Machalí y Graneros). Esto significó un aumento significativo de la población que saturó los servicios, aún cuando existen gran actividad laboral en diferentes rubros: agrícolas (en menor proporción que los casos anteriores), comercial e industrial. Esto podría explicar que en estas comunas la cesantía y la densidad sean las más altas de la provincia y que además aquí donde se concentran la mayor cantidad de pobres (aunque en términos relativos concentran la menor proporción de pobres).

Gráficamente, esto podría mostrarse como sigue:

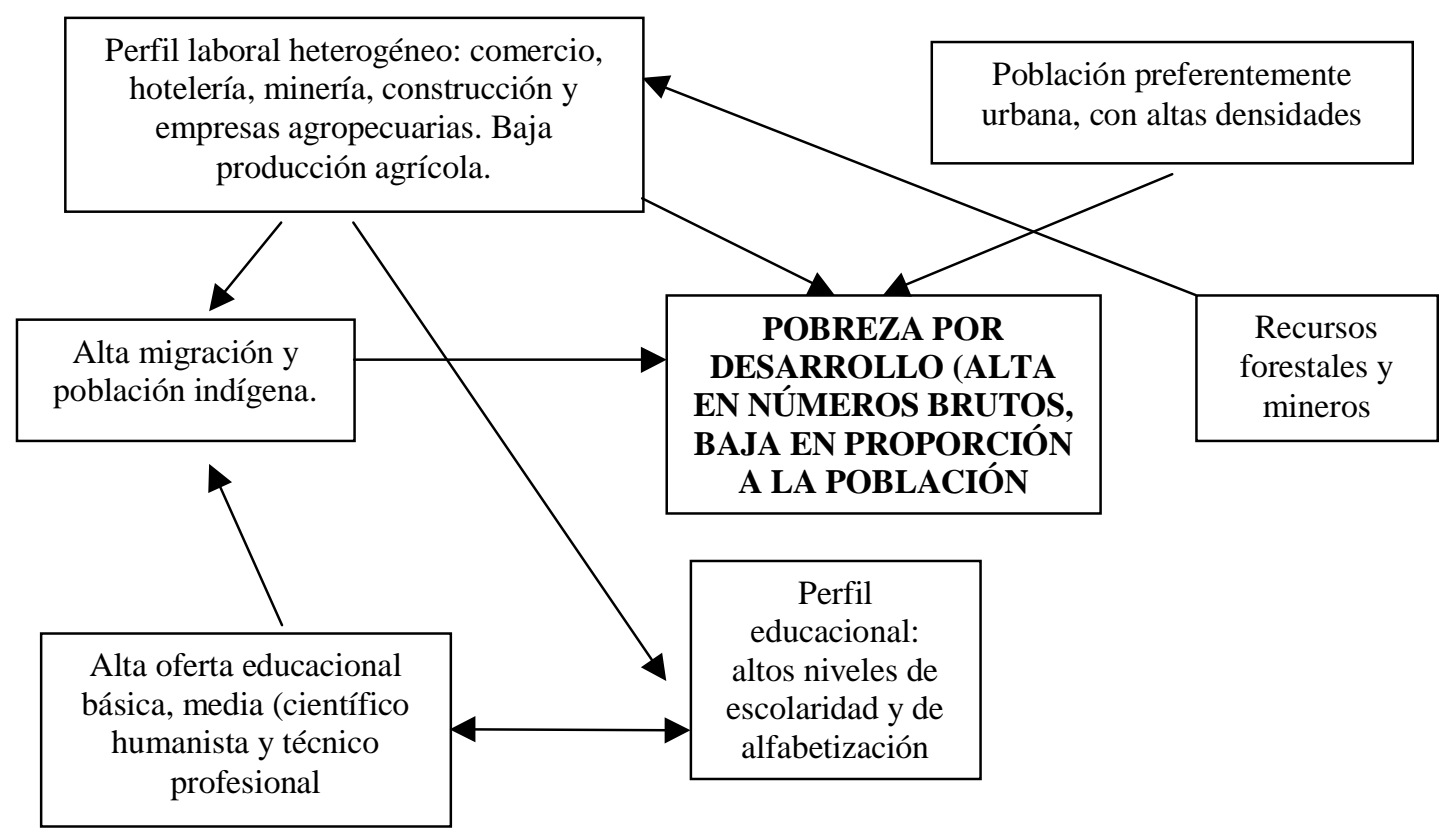


d)

\section{Otros sectores}

Los antecedentes arrojan también cuatro comunas que resultan muy difíciles de analizar como una sola agrupación, ya que presentan características que las diferencian. Estas son San Vicente de Tagua-Tagua, Malloa, Requínoa y Rengo.

San Vicente es una comuna principalmente rural (60\% de su población). Su densidad baja (74) y sólo el $4 \%$ de su población corresponde a minorías étnicas. El 7,1\% son personas migrantes y cuenta con un $44 \%$ de población con necesidades básicas insatisfechas. Esto significa que es una de las 5 comunas de la provincia que posee un porcentaje de personas con NBI inferior al $50 \%$. Casi el 20\% de los hogares de San Vicente son dirigidos por una mujer y prácticamente el $30 \%$ no cuenta con agua potable. Es una comuna con una alta tasa de cesantía al año 1992 $(10,78 \%)$. Las matrículas educacionales son bajas, concentrándose la mayoría de la oferta educacional en la educación preescolar. Sólo el $30 \%$ de la oferta educacional municipal y subvencionada son matrículas de enseñanza básica. La situación es peor en la enseñanza media pues esta comuna no cuenta con ninguna matrícula para la educación secundaria, ni científico-humanista ni técnico-profesional. Esto hace suponer que la mayoría de sus habitantes en edad escolar deben salir de la comuna para terminar sus estudios. El promedio de los años de estudio es 7,1, lo cual es levemente inferior al promedio regional.

Su perfil laboral se caracteriza principalmente por la agricultura aunque en una proporción bastante menor que las comunas que tienen una cantidad similar de población rural (42\%). Los antecedentes del Censo del año 1992 indican además que un porcentaje considerable de su población se dedica a rubros más característicos de sectores urbanos como el comercio (14\%), la industria $(8,27 \%)$, el transporte $(5 \%)$ y la construcción
(4\%). En cuanto al desempleo, la tasa de cesantía es de 7,17.

Los mapas indican que San Vicente es, sin embargo, un sector rico en frutales aunque con una escasa red vial. También hay en ella gran cantidad de empresas familiares y pequeñas empresas de agricultura y silvicultura. También sobresale por tener atractivos culturales considerados en el Plan Maestro de Turismo de la SERPLAC.

Malloa coincide con San Vicente en ser una comuna preferentemente rural (64\%). También coincide en que su población tiene una tendencia que llama la atención de participar en actividades más características de zonas urbanas como el comercio $(8,4 \%)$ y la industria $(16,7 \%)$. De hecho, es la segunda comuna con más participación en este último rubro. A diferencia de San Vicente, su población trabaja en su mayoría en el sector agrícola (52\%) siguiendo la tendencia provincial de trabajar en cultivos en general. En esta comuna hay una cantidad no poco considerable de pequeñas empresas y empresas familiares de agricultura.

El porcentaje de población de Malloa que tiene sus necesidades básicas insatisfechas es de un $54 \%$, lo cual es superior a la proporción de San Vicente. Su oferta educacional está concentrada principalmente en la educación básica (el 90\% de su oferta corresponde a la educación primaria) y, al igual que San Vicente, sus establecimientos no ofrecen matrículas para la educación media ni científico-humanista ni técnico-profesional. El promedio de años de estudio es relativamente bajo para el promedio regional (6,6 años).

Es una comuna, al igual que San Vicente, rica en frutales. Tiene una cantidad importante de atractivos culturales e intersectan en ella la carretera panamericana con la carretera de la fruta .

La comuna de Requínoa también tiene una tendencia prioritaria hacia la población 
rural (55\%). La cantidad de personas con NBI alcanza a un porcentaje del $55 \%$ y un $17 \%$ de sus hogares no cuentan con agua potable. En similitud con las comunas anteriores, si bien la agricultura ocupa un lugar prioritario $(53 \%)$ también existen otras actividades propias del sector urbano: el $16 \%$ de su población se dedica a trabajar en comercio, el 6\% en transporte y el 5\% en industrias. La tasa de cesantía es menor que en las otras comunas $(6,59)$.

Sin embargo, a diferencia de las comunas antes descritas, esta es junto con Rengo, una de las pocas comunas que ofrecen matrículas en enseñanza media y especialmente en educación técnico-profesional. El 75\% de su oferta educacional corresponde a la educación básica, mientras que el $9 \%$ corresponde a matrículas de educación media científicohumanista y el $4,5 \%$ a educación técnicoprofesional.

Rengo es, de estas cuatro comunas, la única que está compuesta mayoritariamente por población urbana (64\%). Además tiene una porcentaje de personas con necesidades básicas insatisfechas relativamente bajo en relación a las otras comunas de la provincia (42\%). Su perfil laboral está más cerca del "sector urbano", ya que sólo el $34 \%$ de su población se dedica a la agricultura, mientras que el $15 \%$ al comercio, el $15 \%$ a la industria, el $5 \%$ al transporte, el $2,8 \%$ a la construcción y el $2 \%$ a la hotelería. Sin embargo, su tasa de cesantía es relativamente alto $(10,16)$

En relación a su perfil educacional, es la comuna que proporcionalmente ofrece más matrículas en educación media científicohumanista $(17,6 \%)$ y, a la vez, la segunda comuna que ofrece más matrículas de educación técnico profesional $(7,75 \%)$. Su promedio de años de estudio es levemente superior a las comunas antes descritas $(7,55)$. Su bien población analfabeta urbana está cerca del promedio provincial (4\%), su po- blación analfabeta rural está cerca de las más altas de la provincia $(9,16 \%)$.

\section{Propuesta Diagnóstica: Indicador Compuesto de Vulnerabilidad Socio-Territorial}

Fruto del trabajo anterior, contemplando los antecedentes recogidos para las tres provincias de la VI Región y utilizando fundamentalmente los datos del Censo de 1992, se propone a continuación una aproximación diagnóstica de carácter cuantitativo.

En general los antecedentes que se recogen de las fuentes secundarias, son datos que utilizados de manera independiente entregan un mirada parcial de la realidad. Es decir es información temática y no territorial.

Sin embargo, como ya se ha planteado lo que interesa a este estudio es utilizar la perspectiva territorial, por cuanto dicho enfoque logra poner en un mismo análisis un conjunto de variables, que articuladas permiten tener una comprensión más integral de la realidad.

Para trabajar la perspectiva territorial, desde el punto de vista de cuantitativo, hemos ordenado toda la información disponible por comunas. Dicho de otra manera cada indicador es utilizado intracomunalmente. Esto con el objeto de igualar todos los espacios territoriales y no privilegiar ninguno de ellos en particular.

De esta manera, podemos tener una mirada de la región, desde una dimensión diferentes, generando igualdad cuantitativamente y con ello dando lugar a apreciaciones cualitativas.

Las variables que hemos escogidos para este efecto son las siguiente: (Datos extraídos del Censo de 1992) 
1.- $\quad$ Grado de Ruralidad

2.- Mujeres Jefes de Hogar

3.- Cesantía

4.- Nivel de Servicios

5.- Tasa de Crecimiento

6.- Nivel de Enseñanza Media

7.- Pobreza. Necesidades Básicas Insatisfechas

Los datos de cada una de estas variables fueron trabajadas en el programa computacional Mapinfo, el cual nos permite poner el conjunto de variables en una acción orientada a definir la realidad integral de cada comuna.

El conjunto de estas variables conforman lo que hemos denominado provisoriamente Indicador de Vunerabilidad Socio-Territorial. Este indicador tiene por objetivo entregar una apreciación integral de lo que ocurre desde el punto de vista de la vulnerabilidad en un territorio determinado. La vulnerabilidad socio-territorial aquí es entendida como aquella situación de riesgo integral en que se encuentra la población de un espacio territorial determinado. Este tipo de vulnerabilidad va más allá de tener sólo un problema de pobreza, sino que además significa que hay problemas en otras áreas, ya sea educación, ya sea disponibidad de servicio, ya sea la cesantía, etc de modo que la situación de vida de la población puede ser aún más grave que visto desde indicadores parciales. Dicha vulnerabilidad además esta representada territorialmente, de modo que permite realizar una comparación intraregional.

De acuerdo al análisis cuantitativo se ha estructurado este indicador en los siguiente niveles. Baja Vulnerabilidad; Vulnerabilidad Media; Alta Vulnerabilidad, y Muy Alta Vulnerabilidad. Estos se encuentran expresados en el siguiente Mapa.

\section{VULNERABILIDAD SOCIO-TERRITORIAL EN LA VI REGION}

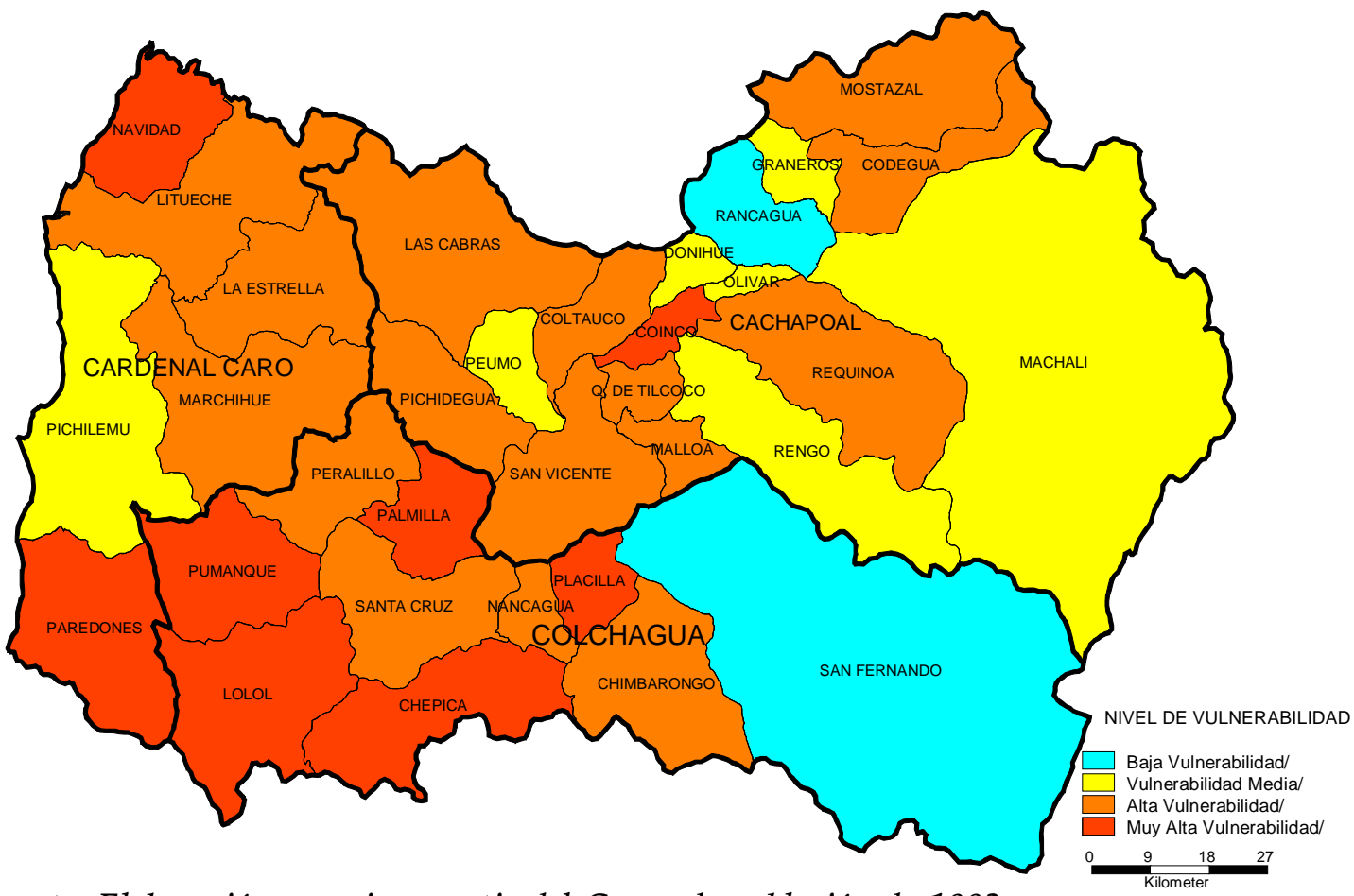

Fuente: Elaboración propia a partir del Censo de población de 1992 
Como se puede observar en el mapa $\mathrm{N}^{\circ} 1$ existe una Muy Alta Vulnerabilidad en las comunas de Paredones, Pumanque, Lolol , Chepica, Navidad, Palmilla, Placilla y Coinco. Por otro lado las comunas con Baja Vulnerabilidad serían Rancagua y San Fernando. El resto de las comunas se encuentra en Media y Alta Vulnerabilidad.

Muchas deducciones son posibles extraer de esta forma de diagnosticar la región. Por lo pronto se podría señalar que el fuerte de acción de los programas sociales desarrollados por las instituciones estudiadas y otras debiera radicarse en las comunas de muy alta y alta vulnerabilidad. Como sí mismo no necesariamente debieran estar abocada a trabajar en las comunas de baja vulnerabilidad.

\section{Conclusiones}

Como se señala más arriba las conclusiones que se presentan a continuación corresponden al análisis de los resultados de las tres provincias de la VI Región. Y estas serían las siguientes.

1. Es posible identificar Polos de Concentración y Desarrollo, que se caracterizan por; una gran concentración de población, predominio urbano, altos índices de escolaridad en comparación con el promedio regional, buenos índices de calidad de vida, perfil laboral con un promedio inferior al regional en el sector agrícola, superior al regional en el sector servicios, comercio y manufactura, mayor diversificación en el perfil laboral en general en comparación con el resto de la región. Adecuada cobertura de servicios públicos tales como, agua, electricidad, obras públicas y salud, y don- de la pobreza más probablemente ligada a la Pobreza Moderna, se caracteriza por sectores marginales urbanos que representan un gran número (en términos absoluto) en comparación con la pobreza total de la región, pero proporcionalmente reducido en relación con las personas que tienen sus Necesidades Básicas Satisfechas en la misma comuna.

Estos Polos de Concentración y desarrollo coinciden en general con las Capitales Provinciales, y con algunas de las ciudades con mayor número de habitantes de la región.

2. Es posible identificar en la dinámica regional que el proceso de concentración y desarrollo que se observa en las capitales provinciales, resulta mayormente acentuado en la capital de la región y con ello en la Provincia de Cachapoal, seguido por San Fernando en la Provincia de Colchagua, y con cierto rezago por Pichilemu en la Provincia de Cardenal Caro. Además la relación urbano - rural, y la proporción de la población con necesidades básicas insatisfechas, se invierte entre un polo y otro, observándose cierta equiparidad en el caso del polo de concentración y desarrollo intermedio, Colchagua - San Fernando. Así mientras en Cardenal Caro, la población rural practicamente dobla a la población urbana y la población con necesidades básicas insatisfechas duplica a la población con necesidades básicas satisfechas, en Cachapoal, la población urbana supera en general a la población rural, y la población con necesidades básicas insatisfechas es inferior proporcionalmente a la pobla- 
ción que tiene sus necesidades básicas satisfechas.

3. Es posible observar que la pobreza ligada a los polos de concentración y desarrollo es más probablemente Pobreza Moderna, y que la que se presenta en los sectores de menor concentración y desarrollo se encuentre ligada a la Pobreza Rural por Atraso.

4. Es posible observar sectores de agricultura tradicional ubicados principalmente en la zona del valle central de las provincias de Cachapoal y Colchagua, donde existe un predominio rural con un perfil laboral preferentemente agrícola, menores índices educacionales, con ciertas concentraciones de poblaciones urbanas lo que da por resultado la coexistencia de Pobreza Moderna con Pobreza por Atraso.

5. También es posible identificar la zona del secano costero e interior, constituido principalmente por la Provincia de Cardenal Caro y algunas comunas de Cachapoal y Colchagua donde coexisten problemas de riego y desgaste del suelo, con altos porcentajes de población rural, bajos índices educacionales y baja densidad poblacional, a lo que se suma la lejanía de la Capital Regional, y las dificultades de acceso vial. En esta zona la pobreza se explica fundamentalmente por el atraso.

6. En la dimensión educacional podemos observar que la infraestructura educacional es en general inadecuada a nivel regional, existiendo una gran escasez de matrículas Científico Humanista y principalmente escasez de matrículas Técnico Profesionales, esto principalmente en los sectores ajenos a los polos de concentración y desarrollo, la zona agrícola tradicional y el secano costero e interior, lo que incide en la escasa transferencia tecnológica para la diversificación productiva, el mejor aprovechamiento de los recursos y del capital social.

7. El Análisis de las Dinámicas Provinciales nos permite observar el proceso circular que retroalimenta la pobreza, donde la baja escolaridad y la agricultura tradicional se autoperpetúan por la escasa transferencia tecnológica, dada la escasa cobertura de la enseñanza media, principalmente Técnico Profesional, y secundariamente Humanista, lo cual limita el nexo necesario para la racionalización y diversificación de los recursos, y trae como efecto secundario pero no despreciable, la permanente migración del capital social mayormente preparado y motivado hacia los polos de concentración y desarrollo. Con ello se saturan los servicios y las posibilidades de mercado laboral en estos últimos acentuando la marginalidad y la Pobreza Moderna.

8. El Análisis de las Dinámicas Provinciales también nos permite constatar que es posible mediante el enfoque territorial y el mismo análisis de dinámicas, focalizar los sectores de mayor rezago educacional y productivo, y las variables que auto reproducen el círculo de la pobreza moderna y por atraso, lo cual sugiere una mayor profundización en el análisis de las dinámicas locales, dentro de los focos de interés, incluyendo el enfoque territorial a fin de perfeccionar las estrategias de intervención orientadas a la superación de la pobreza, teniendo presente la multicausalidad y dependencia del contexto geográfico que tiene el fenómeno. 


\section{Bibliografía}

1.- CEPAL, (1992) Equidad y Transformación Productiva : Un Enfoque Integrado.

2.- CEPAL, (1997) Panorama Social de América Latina.

3.- Chile Barrio (1998) Chile_Barrio. Santiago de Chile.

4.- Estrategias de Desarrollo RegioNAL 2000-2010. CORE

5.- Fosis (1997) Documentos Institucionales

6.- Fosis (1997) Documentos Institucionales

7.- INDAP, (1998) "Hacia el 2000. La experiencia de los programas PRODESAL". 1998

8.- INE, Censo 1992

9.- Kleymeyer, Ch. (1993)Cultural Expression and Grassroots Development. Fundación Interamericana.

10.- KLIKSBERG,B. (1998). Repensando el Estado para el Desarrollo Social: Más allá de Dogmas y Convencionalismos. Proyecto Regional de Naciones Unidas .

11.- KliKsberg,B. (1997). Pobreza. Un tema impostergable. Fondo de Cultura Económica,

12.- Glaser, B., \& Strauss, A. (1967). The discovery of grounded theory. Chicago: Aldene Publishing Company. 13a.- PNUD, (1998) “Desarrollo Humano en Chile - 1998. Las Paradojas de la Modernización" Ed. PNUD

13b.- PNUD, (2000) "Indice de Desarrollo Humano", 2000. PNUD

14.- Memoria Gobierno Regional VI Región 1995-1999

15.- MidePlan, 1998, Encuesta de caracterización Socio-económica Nacional (Casen)

16.- MINSAL-INE . Estadísticas 1999

17.- MINEDUC Estadística. División de Planificación y Presupuesto. 1992

18.- Municipalidad de Rancagua (2000) Boletín informativo Programa Mujeres de Hogar. Rancagua, Chile.

19.- PRIT (1999) n 6 Cd Programa Regional de información Territorial VI Región, SIRIG

20.- PRORURAL “La experiencia de Prorural 1998-1999".

21.- PRODEMU (2000) PRODEMU. Santiago de Chile.

22.- Strauss, A., \& Corbin, J. (1990). Basic of qualitative research. Newbury Park. Ca: Sage.

23.-Valenzuela, R. (2.000) Documento Comité Técnico Regional y Subsidio a la Información CORE.

24.-WORLD BANK

(1997) Worl

Development Indicators 
